\title{
Purificação e caracterização da VDAC de mitocôndrias corticais aviares: identificação de modificações pós-traducionais nas porinas neuronais murinas e aviares ${ }^{1}$
}

\author{
Phelipe Augusto Mariano Vitale ${ }^{2}$, Carla Rossini Crepaldi², Andréa Cristina Tesch ${ }^{2}$, \\ Ricardo de Albuquerque ${ }^{3}$ e Marcelo de Cerqueira César ${ }^{2 *}$
}

\begin{abstract}
Vitale P.A.M., Crepaldi C.R., Tesch A.C., Albuquerque R. \& César M.C. 2012. [Purification and characterization of avian cortical mitochondrial VDAC: identification of post-translational modifications of rat and avian neuronal porins.] Purificação e caracterização da VDAC de mitocôndrias corticais aviares: identificação de modificações pós-traducionais nas porinas neuronais murinas e aviares. Pesquisa Veterinária Brasileira 32(12):1361-1366. Laboratório de Neurociência e Proteômica, Faculdade de Zootecnia e Engenharia de Alimentos, Universidade de São Paulo, Av. Duque de Caxias Norte 225, Pirassununga, SP 13635-900, Brazil. E-mail: mccesar@usp.br

VDAC (voltage-dependent anion channel) is a pore forming protein from outer mitochondrial membrane. It has key functions on energetic metabolism, and cell death and survival. VDAC characterization is important for understanding mitochondrial interactions with cytosolic proteins, such as hexokinase (HK). HK-VDAC interaction supports preferential access to intramitochondrial ATP in neural cells. Brain HK interacts in different ways with VDAC. It results in two HK binding sites (A and B). VDAC isoforms differential metabolic roles may be explained by the presence of post-translational modifications. In this study we purified avian neuronal mitochondrial VDAC1. At same time we showed that VDACs 1 and $2 \mathrm{p} I$ heterogeneity in rat and avian brains is due to phosphorylation. Purified VDAC had a molecular weight of $30 \mathrm{KDa}$. The purified VDAC submitted to phosphorylated protein staining on gel, was dephosphorylated. The knowledge of presence or absence of VDAC phosphorylation is important for understanding the molecular nature basis of A and B HK binding sites in brain mitochondria.
\end{abstract}

INDEX TERMS: VDAC, hexokinase, interactome, phosphorylation, mitochondria, neuron, brain.

RESUMO.- A VDAC é uma porina presente na MME cuja função é crucial no metabolismo energético, sobrevivência e morte celular. A caracterização da VDAC torna-se importante para a compreensão das inter-relações da mitocôndria com os diferentes componentes citosólicos, tais como a HK. A ligação HK-VDAC favorece a utilização do ATP intramitocondrial em células neuronais, a HK cerebral pode interagir de formas diferentes com a VDAC, o que resulta

\footnotetext{
${ }^{1}$ Recebido em 26 de janeiro de 2012.

Aceito para publicação em 20 de setembro de 2012

${ }^{2}$ Departamento de Ciências Básicas, Faculdade de Zootecnia e Engenharia de Alimentos, Universidade de São Paulo (USP), Av. Duque de Caxias Norte 225, Pirassununga, SP 13630-000, Brasil. *Autor para correspondência: mccesar@usp.br

${ }^{3}$ Departamento de Nutrição e Produção Animal, Faculdade de Medicina Veterinária e Zootecnia, USP, Av. Duque de Caxias Norte 225, Pirassununga, SP 13630-000.
}

em diferentes sítios de ligação (sítios A e B). Os variados papéis metabólicos das isoformas da VDAC podem ser explicados pela presença de alterações pós-traducionais. No presente trabalho purificamos a VDAC1 mitocondrial neuronal proveniente de cérebro aviar. Paralelamente, comprovamos que a presença de múltiplas formas das VDACs 1 e 2 em cérebros murino e aviar, seja devida à presença de modificações pós-traducionais, nomeadamente a fosforilação. A proteína isolada apresentou peso molecular de $30 \mathrm{KDa}$. Quando submetida à eletroforese e posteriormente à coloração para a identificação de fosfoproteínas, a mesma mostrou-se desfosforilada. 0 conhecimento da presença, ou ausência de fosforilação das VDACs, reside na importância de estabelecer-se as bases moleculares ligadas à existência de sítios A e B nas mitocôndrias neuronais.

TERMOS DE INDEXAÇÃO: VDAC, hexoquinase, interactôma, fosforilação, mitocôndria, neurônio, cérebro. 


\section{INTRODUÇÃO}

A VDAC (Canal Aniônico Voltagem Dependente) é uma proteína presente na MME (membrana mitocondrial externa), tendo peso molecular entre 29 e $37 \mathrm{KDa}$ (Sorgato \& Moran 1993). A estrutura da VDAC é composta por aproximadamente 280 aminoácidos (Sampson et al. 1997). A VDAC tem a conformação espacial de um $\beta$-barril anfipático, composto por $16 \beta$-folhas. Em anexo ao $\beta$-barril encontra-se a sequência de aminoácidos que compõem a $\alpha$-hélice $N$-terminal (Casadio et al. 2002), a qual ajudaria na fixação da porina à MME. Além desta função sabe-se que $\alpha$-hélice $\mathrm{N}$-terminal pode interagir com outros componentes, tais como o citocromo c, Smac/Diablo e AIF, que são importantes fatores pró-apotóticos. A $\alpha$-hélice N-terminal da VDAC é vital para o estabelecimento da ligação Hexoquinase (HK)-VDAC sendo, portanto vital para as funções da porina (Pastorino et al. 2002).

É descrito na literatura científica que a HK cerebral pode interagir de formas diferentes com a VDAC, o que resulta em diferentes sítios de ligação. Cada sítio apresenta certa diferença em relação ao outro, o que pode ser demonstrado através do tratamento experimental das mitocôndrias com glicose-6-fosfato (G-6-P). Por meio deste tratamento demonstrou-se a existência de dois sítios de ligação: o sítio A e o sítio B (KABIR e WILSON 1994). 0 sítio A mostrou-se susceptível ao tratamento com G-6-P, a qual desliga a HK do mesmo. Entretanto, a hexoquinase ligada ao sítio B mostrou-se refratária a esse tratamento. Portanto, a proporção entre o sítio tipo A e o sítio tipo B varia entre as diferentes espécies animais, sendo de aproximadamente 90:10, 60:40, 40:60, e 20:80 nas mitocôndrias cerebrais de rato, coelho, bovino e humano, respectivamente (Cerqueira César \& Wilson 2002). De acordo com Hutny \& Wilson (2000) as bases moleculares que geram a existência dos sítios de ligação consistem na presença de diferentes fosfolipídios da membrana mitocondrial externa.

Relatou-se que é possível apenas religar a hexoquinase desligada do sítio A. Por outro lado, o sítio B é intrinsecamente incapaz de permitir que uma vez a hexoquinase liberada, seja religada. 0 fato de não se realizar a religação ao sítio B suportaria a suposição de que somente o sítio A é importante fisiologicamente na relação de liberação/ religação da $(\mathrm{HK})$, o que poderia acontecer em resposta à necessidade energética do cérebro. Foi sugerido que o sítio B deverá agir como reservatório de hexoquinase, liberando a enzima para o sítio A em situações de alta demanda energética da célula (Golestani et al. 2007).

São conhecidas três isoformas da VDAC (VDAC1, VDAC2 e VDAC3) em mamíferos (Sampson et al. 1998). A célula neuronal aviar apresenta uma expressão mais elevada da VDAC1, em relação às outras duas isoformas (Poleti et al. 2010). Valores elevados no nível de VDAC 1 e baixos níveis de VDAC 2 foram observados em um grupo de pacientes epiléticos fármaco-resistentes, o que pode estar relacionado com a diminuição da produção e translocação de ATP mitocondrial, sendo que esta diminuição causa falha energética e agrava a disfunção mitocondrial (Jiang et al. 2007).

Adicionalmente Poleti et al. (2010) observaram que a VDAC 1 e VDAC 2 apresentam heterogeneidade de pontos isoelétricos. A VDAC1 foi dividida em dois spots nas células neuronais murinas e bovinas e em três spots em células neuronais aviares. A VDAC2 foi separada em três, cinco, e dois spots em células neuronais de murino, bovino e aviar, respectivamente (Poleti et al. 2010). Outro aspecto diferencial entre neurônios aviar e murino, reside no fato de que nos primeiros a VDAC faz parte de mais complexos proteicos (5), que nos últimos (1) indicando uma cinética diferenciada de montagem (Crepaldi et al. 2011).

As alterações pós-traducionais das VDACs compreendem acetilação de lisina e fosforilação de serina, treonina e tirosina (Shoshan-Barmatz et al. 2010). Neste sentindo a VDAC 1 apresenta fosforilação de duas serinas, Ser-12 e Ser-136. Por outro lado, a VDAC 2 apresenta fosforilação da Tyr-237 e a VDAC3 da Thr-33 e Ser-241 (Distler et al. 2007).

Assim, em todas as três isoformas da VDAC os resíduos de aminoácidos fosforilados são acessíveis à modificação mediada por proteínas quinases, e fosfatases localizadas no espaço citosólico e intermembrana, o que pode explicar os diferentes papéis metabólicos das isoformas da VDAC. No presente trabalho purificamos a VDAC1 e comprovamos ser a heterogeneidade das VDACs 1 e 2 em cérebros murino e aviar devida à presença de fosforilação.

\section{MATERIAL E MÉTODOS}

Os cérebros aviares foram retirados e pesados logo após o abate dos animais no matadouro escola da USP, Campus de Pirassununga. Por sua vez, os cérebros de rato, foram obtidos após eutanásia de animais provenientes do biotério da Universidade Camilo Castelo Branco, campus Descalvado (SP). Para cada extração mitocondrial foram utilizados 3 cérebros íntegros, tanto de procedência murina como aviar. Após a remoção, o material biológico foi imediatamente imerso em nitrogênio líquido.

0 isolamento mitocondrial foi realizado conforme descrito por Crepaldi et al. (2011), seguindo o mesmo procedimento, tanto para o material de procedência murina, quanto para o de procedência aviar. As proteínas totais foram determinadas pelo kit BCA Protein Assay Kit (código 23225, Thermo Scientific) utilizando como padrão uma solução de albumina bovina. As mitocôndrias isoladas foram armazenadas em nitrogênio líquido.

Após o isolamento mitocondrial, a amostra foi submetida à solubilização e à Cromatografia pelos métodos descritos por De Pinto et al. (1987) e Gincel et al. (2000). As mitocôndrias (5mg/ml) sofrem choque osmótico pela ação do tampão Tris-EDTA $(10 \mathrm{mM}$ Tris-HCL, $1 \mathrm{mM}$ EDTA, 0,25mM PMSF e $0,5 \mu \mathrm{g} / \mathrm{ml}$ leupeptina, $\mathrm{pH}$ $7,0)$ e são centrifugadas por 30 minutos a $15100 x g$. As membranas foram solubilizadas pelo tampão de solubilização (3\% Triton $\mathrm{X}-100,10 \mathrm{mM}$ Tris-HCL, 1mM EDTA, 0,25mM PMSF e 0,5 $\mu \mathrm{g} / \mathrm{ml}$ leupeptina, $\mathrm{pH} 7,0$ ). Em seguida o pellet foi mantido a $25^{\circ} \mathrm{C}$ por 30 minutos para que o tampão de solubilização separasse a VDAC da membrana mitocôndrial externa. Após este período, a amostra foi centrifugada a $15100 x g$ durante 30 minutos. 0 sobrenadante foi submetido à cromatografia.

A coluna cromatográfica era composta por uma resina de $\mathrm{Hi}$ droxiapatita Bio-Gel HTP (BIO-RAD) e Celite 535 (codigo 22138, Fluka) na proporção de 2:1. A amostra foi aplicada diretamente na coluna. A eluição foi realizada com o tampão de solubilização. Os eluatos foram concentrados utilizando acetona gelada. A amostra foi tratada com acetona gelada na proporção de 1:1 e centrifugada a $15100 x g$ por 30 minutos. Após este processo, o sobrenadante foi retirado e o pellet foi ressuspenso em tampão de solubilização. 
O preparo do gel segue a técnica descrita por Laemmli (1970). A corrida eletroforética foi mantida a uma voltagem de $200 \mathrm{~V} \mathrm{du}$ rante 60 minutos. Ao final da corrida, os géis foram corados por 1 hora com azul de Coomassie (0,1\% coomassie blue R-250, 10\% ácido acético e $40 \%$ metanol) e descorados overnight em solução descorante (40\% Metanol, 10\% Ácido Acético). As imagens dos géis 2-DE foram obtidas usando o ImageScanner PowerLook 1120 e analisados pelo software Image Quant -TL (GE Healthcare).

Para a realização do gel 2-D as mitocôndrias foram diluídas em solução de reidratação "DeStreak" (Uréia 7M, Thiouréia 2M, CHAPS $2 \%$ e azul de bromofenol 0,002\%), acrescentado de 0,5\% de tampão de IPG pH 3-10, 20mM DTT e 1\% Inibidores de Protease.

Esta solução foi utilizada para uma reidratação passiva das tiras de IPG (13cm; pH 3-10 L) no Immobiline Drystrip Reswelling Tray durante 14 horas à temperatura ambiente. A primeira dimensão (Focalização Isoelétrica - IEF) foi realizada no aparelho Ettan IPGphor à temperatura de $20^{\circ} \mathrm{C}$ por aproximadamente $5 \mathrm{~h}$, com uma voltagem inicial de $500 \mathrm{~V}$ e em seguida, aplicando-se um gradiente de 1000 à $8000 \mathrm{~V}$ tendo no fim uma voltagem estável de $8000 \mathrm{~V}$, com um limite de corrente de $75 \mathrm{~mA} /$ tira.

Para a segunda dimensão, as tiras foram equilibradas por 15 minutos em Tampão de Equilíbrio I e 15 minutos, em Tampão de Equilíbrio II sob leve agitação (Tampão de Equilíbrio I: 6M ureia, $75 \mathrm{mM}$ Tris-HCl pH 8.8, 29,3\% Glicerol, 2\% SDS, 0,002\% azul de bromofenol, 65mM DTT; Tampão de Equilíbrio II: 6M uréia, $75 \mathrm{mM}$ Tris-HCl pH 8.8, 29,3\% Glicerol, 2\% SDS, 0,002\% azul de bromofenol, 197mM Iodoacetamida) e em seguida, sobrepostas ao gel de acrilamida (SDS-PAGE $12 \%)$ ( $18 \times 16 \times 0.15 \mathrm{~cm})$. A tira foi fixada sobre o gel com uma solução selante de agarose $(25 \mathrm{mM}$ Tris base, $192 \mathrm{mM}$ glicina, $0,1 \%$ SDS, 0,5\% agarose e $0,002 \%$ de azul de bromofenol). 0 tampão de corrida utilizado foi o de Laemmli (1970) (1x concentrado para o anodo e $3 x$ concentrado para o cátodo). Para a corrida eletroforética foi mantida uma corrente de $25 \mathrm{~mA} /$ gel a uma voltagem inicial de $90 \mathrm{~V}$ por 30 minutos e posteriormente aumentada para $250 \mathrm{~V}$ por $6 \mathrm{~h}$.

Ao final da corrida, os géis foram submetidos à detecção de proteínas fosforiladas via coloração pelo Methyl green através do kit GelCode Phosphoprotein Staining kit (Thermo Scientific). Após esta etapa o gel foi escaneado para obtenção da imagem das proteínas fosforiladas e em seguida submetido a sucessivas lavagens com água destilada e corado por $1 \mathrm{~h}$ com azul de Coomassie (0,1\% coomassie blue R-250,10\% ácido acético e $40 \%$ metanol) e descorados overnight em solução descorante (40\% Metanol, 10\% Ácido Acético), com o propósito de visualizar o perfil eletroforético total.

Para a realização do blotting, após a eletroforese, os géis foram transferidos à $80 \mathrm{~V}, 400 \mathrm{~mA}$ durante $1: 40 \mathrm{~h}$ para uma membrana de nitrocelulose com poros de 0,45 $\mu \mathrm{m}$, utilizando a unidade de transferência TE 62 (GE Healthcare) e o sistema de tampão de Towbin et al. (1979). As membranas foram bloqueadas pela incubação overnight à $4^{\circ} \mathrm{C}$ em tampão TBS $(20 \mathrm{mM}$ Tris, $0,5 \mathrm{M} \mathrm{NaCl}$, pH 7.5) contendo $5 \%$ de leite em pó desnatado e $1 \%$ gelatina. As membranas foram incubadas com anticorpos primários anti-VDAC1 e secundários conjugados com peroxidase (Bio-Rad). Os anticorpos foram diluídos em tampão TBS contendo 1\% gelatina. Em seguida, a quimiluminescência foi revelada em filme de raio-X no Hospital Veterinário da Faculdade de Medicina Veterinária e Zootecnia da Universidade de São Paulo - campus de Pirassununga.

\section{RESULTADOS}

O protocolo de purificação escolhido envolve a solubilização da proteína com detergente, seguido por cromatografia
(De Pinto et al. 1987). No caso, a membrana mitocondrial externa foi dissolvida com um detergente não iônico, o Triton X-100. Na presença deste detergente, ou qualquer outro que contenha uma cauda de polioxietileno ligada a um grupo hidrofóbico, a VDAC solubilizada apresenta o aspecto peculiar de ser eluída sem interação com a fase estacionária (Shoshan-Barmatz et al 2010). Esta característica peculiar permite a purificação da VDAC em uma única etapa cromatográfica (Fig.1). A Figura 1 demonstra que a VDAC foi eluída principalmente na fração cromatográfica número 4, demonstrando a existência de uma banda de peso molecular compatível com o da VDAC, de 30KDa. A confirmação foi realizada via Immunoblotting com anticorpos monoclonais anti-VDAC 1 (Fig.2).

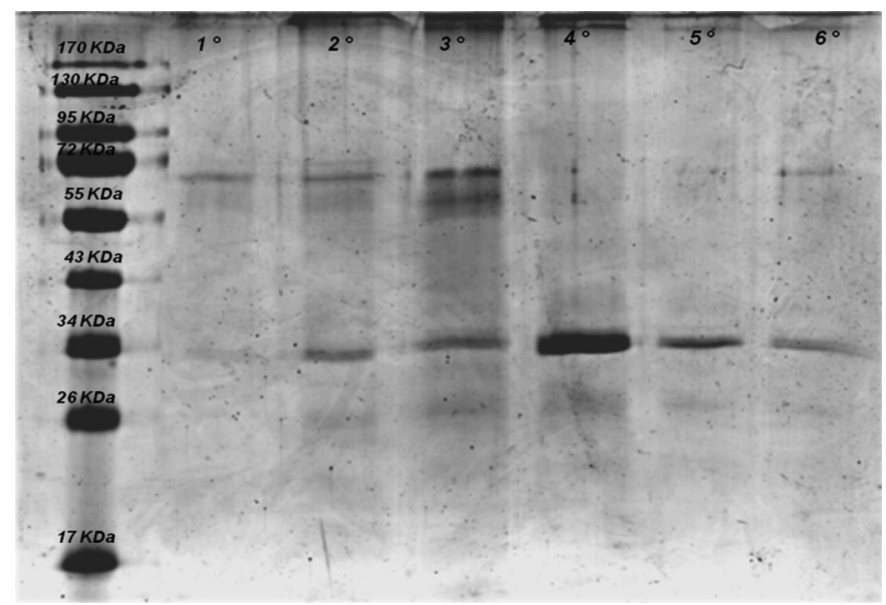

Fig.1. Gel SDS PAGE corado com coomassie demonstra os diferentes perfis protéicos de cada eluato, sendo o 4, aquele que apresenta a maior concentração da VDAC mitocondrial neuronal aviar.

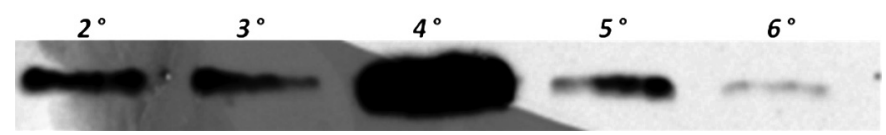

Fig.2. Western blotting realizado com anticorpo anti-VDAC 1 confirmando uma maior intensidade da VDAC1 no quarto eluato.

Visando à confirmação da presença de fosforilação das VDACs, géis Bi-Dimensionais de suspensões mitocondriais, contendo todas as proteínas desta organela, foram submetidos à detecção de proteínas fosforiladas pelo método de coloração com Methyl green. Os reagentes deste kit hidrolisam a ligação fosfoéster, fosfoserina e fosfotreonina, portanto a fosfotirosina não é hidrolisada e não pode ser detectada.

A Figura 3 apresenta um gel de proteínas mitocondriais de cérebro aviar. Publicação anterior do laboratório havia identificado os spots assinalados via Western blotting e espectrometria de massas (Poleti et al. 2010),em um padrão eletroforético de alta reprodutividade. Tendo confirmado a identidade dos spots como VDACs 1 (spots a, b, d) e 2 (spots c, e), por sobreposição em relação a um gel padrão, procedemos à execução de géis, para, com utilização de coloração específica para proteínas fosforiladas, confirmar a existência de fosforilação (ou não) das VDACs. 
Neste sentido, a Figura 4 apresenta um gel de proteínas mitocondriais fosforiladas de cérebro aviar, no qual, também por sobreposição em relação ao gel da figura 3, detectou-se a fosforilação de todas as isoformas da VDAC 1 (a, b, d) e da VDAC2 (c, e), confirmando ser a heterogeneidade de pontos isoelétricos das mesmas devida a esta modificação pós traducional. Foram detectadas três isoformas da VDAC1 (a, b, d) e duas isoformas da VDAC 2 (c, e) fosforiladas.

Por sua vez, a Figura 5 apresenta um gel de proteínas mitocondriais de cérebro murino corados com coomassie blue, tendo sido a identidade dos spots confirmada por sobreposição em relação a um gel padrão, no qual confirmou se a existência das VDACs 1 e2. Os spots a e b foram identificados como VDAC1 e os spots c, d, e como VDAC2

Analogamente ao procedimento efetuado com cérebro aviar, a Figura 6 apresenta um gel de proteínas mitocondriais fosforiladas de cérebro murino, onde confirmou-se a fosforilação da isoformas $1(\mathrm{a}, \mathrm{b})$ e 2 da VDAC (c, d, e).

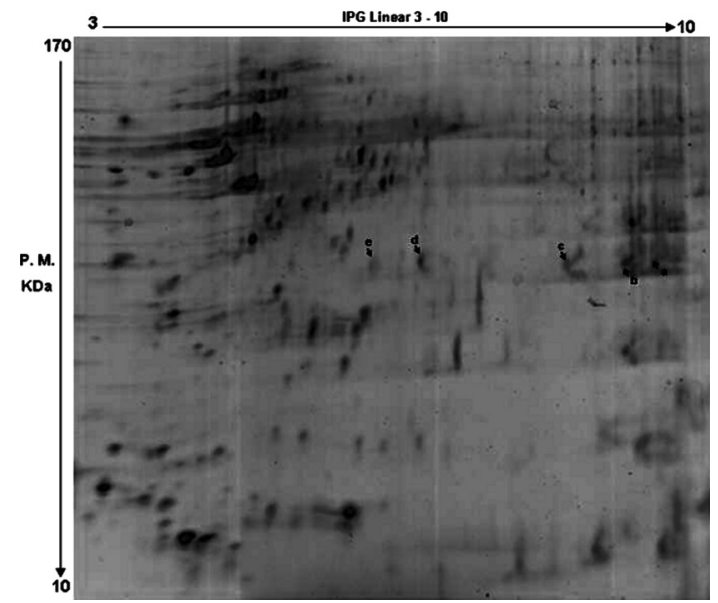

Fig.3. Gel 2DE de proteínas mitocondriais de cérebro aviar. As letras representam VDAC 1 (a, b, d) e a VDAC 2 (c. e).

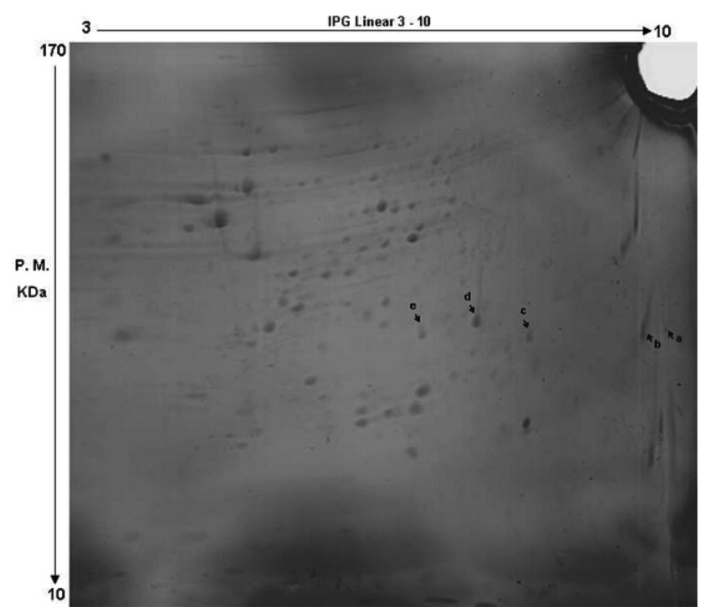

Fig.4. Gel 2DE de proteínas mitocondriais fosforiladas de cérebro aviar. Os spots sinalizados foram detectados por sobreposição (Matches) com o gel correspondente (Fig.3) no software ImageMaster Platinum. As letras representam $\operatorname{VDAC} 1(\mathrm{a}, \mathrm{b}, \mathrm{d})$ e a VDAC 2 (c, e).

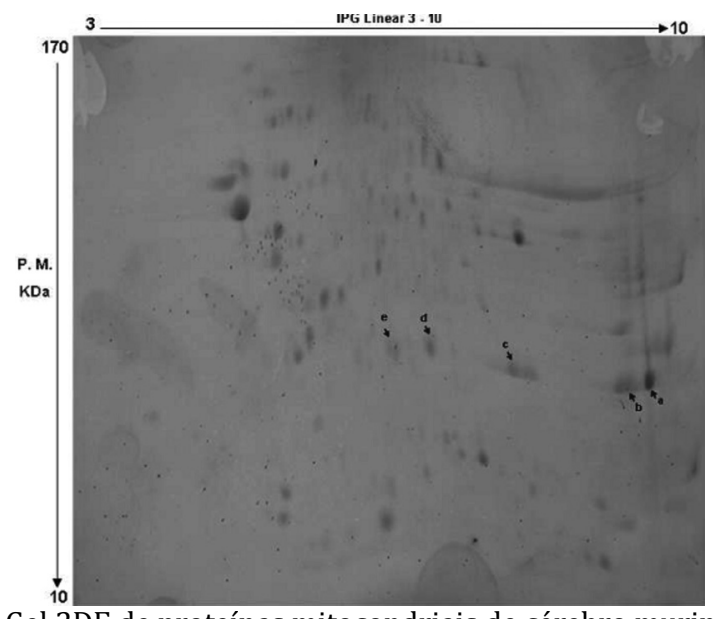

Fig.5. Gel 2DE de proteínas mitocondriais de cérebro murino. . As letras representam VDAC 1 (a, b) e a VDAC 2 (c, d, e).

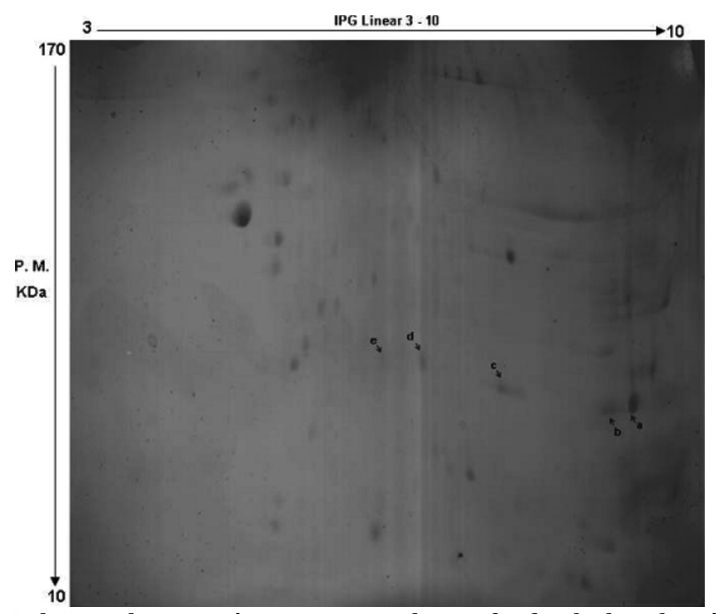

Fig.6. Gel 2DE de proteínas mitocondriais fosforiladas de cérebro murino. Os spots sinalizados foram detectados por sobreposição (Matches) com o gel correspondente (Fig.5) no software ImageMaster Platinum. As letras representam VDAC $1(a, b)$ e a VDAC 2 (c, d, e).

\section{DISCUSSÃO}

Nos últimos anos, a VDAC tem adquirido destaque nos estudos relacionados ao papel das mitocôndrias no processo de apoptose. Desta maneira, a obtenção de dados relativos às modificações pós-traducionais, principalmente os relativos à fosforilação tornam-se importantes no melhor entendimento das interações da VDAC com outras proteínas, especialmente a hexoquinase (Pastorino et al. 2005, Crepaldi et al. 2011).

Vários protocolos diferentes foram desenvolvidos para a purificação da VDAC de tecidos, células ou isolamentos mitocondriais. Porém este trabalho é o primeiro a purificar a VDAC1 de mitocôndrias aviares neuronais (Fig.1 e 2).

Não foi possível confirmar que a VDAC2 tenha sido purificada pela utilização desta metodologia cromatográfica, pois como descrito por Poleti et al. (2010) o anticorpo anti-VDAC 2 foi gerado utilizando-se um polipeptídeo sintético correspondendo à sequência de aminoácidos 120 a 132 da VDAC2 humana. Este anticorpo, contudo apresentou imuno-reatividade cruzada com a VDAC1 de mitocôndrias 
aviares e murina, fato novamente constatado com a VDAC purificada.

Neste trabalho optamos por utilizar a técnica de purificação desenvolvida por De Pinto et al. (1987), por tratar-se de metodologia consagrada e de resultados satisfatórios. Contudo os resultados obtidos durante o estudo mostraram que a VDAC purificada pela metodologia acima não apresentou qualquer grau de fosforilação no gel SDS-PAGE corado com o método de coloração de fosfoproteínas.

Esta observação deve-se, possivelmente ao fato de que a fosforilação confere uma menor hidrofobicidade a uma porção das VDACs. Essas VDACs fosforiladas não seriam purificadas pelo presente metódo. A metodologia utilizada caracteriza-se por isolar proteínas hidrofóbicas. Acreditamos, portanto, estarmos purificando as VDACs não fosforiladas. Estamos desenvolvendo uma metodologia visando à purificação das porções não hidrofóbicas na atualidade

No presente estudo, isoformas da VDAC provenientes de suspensões mitocondriais, apresentaram diversos pontos isoelétricos nos géis que foram submetidos à coloração tradicional por Coomassie (Fig.3 e 5) e na detecção de proteínas fosforiladas pelo método de coloração específico para fosfoproteínas (Fig.4 e 6).

Desta forma, é possível afirmar por sobreposição dos géis que a heterogeneidade nos pontos isoelétricos das isoformas das VDACs seja decorrente primariamente da fosforilação e não de outras alterações pós-traducionais.

Segundo Distler e colaboradores, a existência de múltiplos sítios de fosforilação nas três isoformas da VDAC pode refletir as diferenças funcionais das isoformas (Distler et al. 2007). Neste sentindo, uma das importâncias da fosforilação reside no controle da saída do citocromo $\mathrm{C}$ da mitocôndria através da sua interação com a VDAC (Banerjee \& Ghosh 2006 ). Demonstrou-se que alterações no tamanho dos poros da VDAC em mitocôndrias cerebrais de murinos após interação com Bax e tBid são controlados através da fosforilação da VDAC (Distler et al. 2007). Pastorino e colaboradores demonstraram que o GSK3B (Glycogen Synthase Kinase 3B) fosforila a VDAC em Thr 51 rompendo a ligação da HK-II com a mesma. Uma mutação dessa Thr53 para alanina foi capaz de abolir a capacidade da GSK3B de fosforilar a VDAC, preservando a interação HK-VDAC (Pastorino et al. 2005).

No tecido nervoso a HK está ligada às mitocôndrias através do complexo HK-VDAC, o que favorece a utilização do ATP intramitocondrial no processo de fosforilação da glicose em G6P (glicose-6-fosfato) (Crane \& Sols 1954, Beltrandel Rio \& Wilson 1992, Cerqueira César \& Wilson 1995, 1998, 2002). Esse acoplamento funcional da HK ao ATP intramitocondrial está diretamente relacionado com a proporção de sítios A e B nas diferentes espécies (Cerqueira César \& Wilson 2002).

A hexoquinase é liberada do sítio tipo A de mitocôndrias cerebrais na presença de glicose-6-P; a enzima ligada aos sítios B permanece complexada. A HK ligada aos sítios $B$ não apresenta seletividade pelo ATP intramitocondrial, bem como uma menor sensibilidade a inibição pela G-6-P (Cerqueira César \& Wilson, 2002).

O conhecimento da presença, ou ausência de fosforilação das VDACs, reside na importância de estabelecerem-se as bases moleculares ligadas à existência de sítios A e B nas mitocôndrias neuronais.

Agradecimentos.- Esse projeto teve suporte da Fundação de Amparo à Pesquisa do Estado de São Paulo (Proc. 2010/05560-6). Carla Rossini Crepaldi é bolsista FAPESP (2009/06687-2). Material biológico foi fornecido pela Globoaves.

\section{REFERÊNCIAS}

Banerjee J. \& Ghosh S. 2006. Phosphorylation of rat brain mitochondrial voltage- dependent anion as a potential tool to control leakage of cytochrome c. J. Neurochem. 98(3):670-676.

BeltrandelRio H. \& Wilson J.E. 1992. Interaction of mitochondrially bound rat brain hexokinase with intramitochondrial compartments of ATP generated by oxidative phosphorylation and creatine kinase. Arch. Biochem. Biophys. 299(1):116-124.

Casadio R., Jacoboni I., Messina A. \& Pinto V. De. 2002. A 3D model of the voltage-dependent anion channel (VDAC). FEBS Letters 520:1-7.

Cerqueira César M. \& Wilson J.E. 2002. Functional characteristics of hexokinase bound to the type A and type B sites of bovine brain mitochondria. Arch. Biochem. Biophys. 397(1):106-112.

Cerqueira César M. \& Wilson J.E. 1995. Application of a double isotopic labeling method to a study of the interaction of mitochondrially bound rat brain hexokinase with intramitochondrial compartments of ATP generated by oxidative phosphorylation. Arch. Biochem. Biophys. 324(1):914.

Cerqueira César M. \& Wilson J.E. 1998. Further studies on the coupling of mitochondrially bound hexokinase to intramitochondrially compartmented ATP, generated by oxidative phosphorylation. Arch. Biochem. Biophys. 350(1):109-117.

Crepaldi C.R., Munin F.S., Vitale P.A.M. \& César M.D.C. 2011. Análise interactômica da VDAC (voltage-dependent anion selective channel) nos cérebros aviar, bovino e murinho. Pesq. Vet. Bras. 31(11):1031-1038.

De Pinto V., Prezioso G. \& Palmieri F. 1987. A simple and rapid method for the purification of the mitochondrial porin from mammalian tissues. Biochim. Biophys. Acta 905(2):499-502.

Distler A.M., Kerner J. \& Hoppel C.L. 2007. Post-translational modifications of rat liver mitochondrial outer membrane proteins identified by mass spectrometry. Biochim. Biophys. Acta 1774(5):628-636.

Gincel D., Silberberg S.D. \& Shoshan-Barmatz V. 2000. Modulation of the voltage- dependent anion channel (VDAC) by glutamate. J. Bioenerg. Biomembr. 32(6):571-583.

Golestani A., Ramshini H. \& Nemat-Gorgani M. 2007. A study on the two binding sites of hexokinase on brain mitochondria. BMC Biochem. 8:20.

Hutny J. \& Wilson J.E. 2000. Further studies on the role of phospholipids in determining the characteristics of mitochondrial binding sites for type I hexokinase. Acta Biochim. Pol. 47(4):1045-1060.

Jiang W., Du B., Chi Z., Ma L., Wang S., Zhang X., Wu W., Wang X., Xu G. \& Guo C. 2007. Preliminary explorations of the role of mitochondrial proteins in refractory epilepsy: some findings from comparative proteomics. J. Neurosci. Res. 85(14):3160-3170.

Kabir F. \& Wilson J.E. 1994. Mitochondrial hexokinase in brain: coexistence of forms differing in sensitivity to solubilization by glucose-6-phosphate on the same mitochondria. Arch. Biochem. Biophys. 310(2):410-416.

Laemmli U.K. 1970. Cleavage of structural proteins during the assembly of the head of bacteriophage T4. Nature 227:680-685.

Lai J.C.K. \& Clark J.B. 1979. Preparation of synaptic and nonsynaptic mitochondria from mammalian brain. Methods Enzymol. 55:51-60.

Pastorino J.G., Hoek J.B. \& Shulga N. 2005. Activation of glycogen synthase kinase 3 beta disrupts the binding of hexokinase II to mitochondria by phosphorylating voltage dependent anion channel and potentiates chemotherapy-induced cytotoxicity. Cancer Res. 65(22):10545-10554.

Pastorino J.G., Shulga N. \& Hoek J.B. 2002. Mitochondrial binding of hexokinase II inhibits Bax-induced cytochrome c release and apoptosis. J. Biol. Chem. 277(9):7610 -7618. 
Poleti M.D., Tesch A.C., Crepaldi C.R., Souza G.H.M.F., Eberlin M.N. \& Cerqueira César M. 2010. Relationship between expression of voltage-dependent anion channel (VDAC) isoforms and type of hexokinase binding sites on brain mitochondria. J. Mol. Neurosci. 41(1):48-54.

Crane R.K., \& Sols A. 1954. The non-competitive inhibition of brain hexokinase by glucose-6-phosphate and related compounds. J. Biol. Chem. 210(2):597-606.

Sampson M.J., Lovell R.S. \& Craigen W.J. 1997. The murine voltage-dependent anion channel gene family. J. Biol. Chem. 272(30):18966-18973.

Sampson M.J., Ross L., Decker W.K. \& Craigen W.J. 1998. A novel isoform of the mitochondrial outer membrane protein VDAC3 via alternative spli- cing of a 3-base exon: functional characteristics and subcellular localization. J. Biol. Chem. 273(46):30482-30486.

Shoshan-Barmatz V., Pinto V. De, Zweckstetter M., Raviv Z., Keinan N. \& Arbel N. 2010. VDAC, a multi-functional mitochondrial protein regulating cell life and death. Mol. Aspects Med. 31(3):227-285.

Sorgato M.C., Moran O. \& Pedersen P.L. 1993. Channels in mitochondrial membranes: knowns, unknowns, and prospects for the future. Crit. Rev. Biochem. Mol. Biol. 28(2):127-171.

Towbin H., Staehelin T. \& Gordon J. 1979. Electrophoretic transfer of proteins from polyacrylamide gels to nitrocellulose sheets: Procedure and some applications. Proc. Natl Acad. Sci. USA 76(9):4350-4354. 